\title{
Indices de Trauma: Prediccion del Desenlace
}

\author{
${ }_{1}^{1}$ Silvia Inés Guerrero Macías, ${ }^{2}$ Juan Paulo Serrano Pastrana, ${ }^{3}$ Dairon Gelves Lizcano, ${ }^{4}$ Laura Juliana Delgado Mateus
}

\section{RESUMEN}

Introduccion: Los índices de severidad del trauma son sistemas para clasificar y codificar lesiones, describen objetivamente las condiciones de cada paciente, indicando aquellos con lesiones de mayor severidad, la probabilidad de sobrevida y ayudando a prever desenlaces mórbidos.

Objetivo: Establecer cual índice de severidad predice mejor la morbilidad y mortalidad de los pacientes con trauma penetrante múltiple.

Diseño del estudio: Estudio prospectivo, observacional, de cohorte. En un periodo de 13 meses, se recopilaron los pacientes que ingresaron por trauma penetrante múltiple del Hospital Universitario de Santander. Evaluamos la relacion de los índices de severidad (RTSc, ISS, TRISS) con los desenlaces mediante curvas de regresion logística y la predicción de la mortalidad de cada uno según la sensibilidad, especificidad y la curva ROC.

Resultados: Se incluyeron 111 pacientes, $72 \%$ ingresaron por heridas por arma corto-punzante (HACP) y $28 \%$ por arma de fuego (HPAF). La mortalidad fue del 9.9\%, siendo mayor en las HPAF (23 vs 5\%). En los pacientes con HACP, el área bajo la curva (ROC) para el RTSc fue 0.225 , para el TRISS 0.148 y para el ISS 0.68. En el grupo con HPAF, el área bajo la curva para el RTSc fue 0.247, para el TRISS 0.190 y para el ISS 0.735 .

Conclusiones: EI ISS presentó el valor discriminatorio más significativo, siendo un factor pronóstico adaptable en nuestro medio para evaluar el desenlace (mortalidad). La reanimación inicial previa al ingreso de los pacientes a los centros de atención medica afecta el valor predictivo del RTSc y TRISS.

Palabras claves: Indice anatomico, Indice de severidad, Indice fisiologico, ISS, Mortalidad, RTS, Trauma penetrante, TRISS.

\footnotetext{
${ }^{1}$ Cirujana General, ${ }^{2}$ Cirujano General, Subgerente de Servicios Quirúrgicos, ${ }^{3}$ Medico General Servicio de Cirugía ${ }^{4}$ Medico General

${ }^{1}$ Hospital Universitario de Santander, Bucaramanga, Colombia

${ }^{2}$ Docente Departamento de Cirugía, Hospital Universitario de Santander, General Hospital Universitario de Santander Bucaramanga, Colombia

${ }^{3}$ General Hospital Universitario de Santander, Bucaramanga Colombia

${ }^{4}$ Servicio de Urgencias de Cirugia General, Clinica Guane ESE, Bucaramanga, Colombia
}

Corresponding Author: Laura Juliana Delgado Mateus Medico Servicio Social Obligatorio, Clínica Guane ESE Bucaramanga, Colombia, e-mail: lauradelgado.md@gmail. com
How to cite this article: Macías SIG, Pastrana JPS, Lizcano DG, Mateus LJD. Indices de Trauma: Prediccion del Desenlace. Panam J Trauma Crit Care Emerg Surg 2015;4(3):172-179.

\section{Source of support: Nil}

Conflict of interest: None

\section{ABSTRACT}

Introduction: The severity of trauma indexes are systems to assess, classify and code injuries. They describe objectively the conditions of each patient, indicating those with more severe injuries, the probability of survival and help to provide morbid outcomes.

Objective: To establish the severity trauma index which best predicts morbidity and mortality in patients with multiple penetrating trauma.

Study design: Prospective, observational, cohort study. During 13 months, we collected all patients admitted for multiple trauma penetrating in the Universitary Santander's Hospital. We evaluated the association between the severity trauma indexes (RTSc, ISS, TRISS) and the outcomes, using logistic regression curves and the mortality prediction of each one, according to the sensitivity, specificity and ROC curve.

Results: One hundred and eleven patients were included, $72 \%$ were admitted for stab wounds (SW) and $28 \%$ by gunshot wounds (GW). Mortality was $9.9 \%$, more common in the SW group (23 vs 5\%). In the SW group, the area under the curve (ROC) for RTSc was 0.225 , for TRISS 0.148 and ISS 0.68 . In the group with $\mathrm{GW}$, the area under the curve (ROC) was 0.247 for RTSc, 0.190 for TRISS and 0.735 for ISS.

Conclusion: The ISS had the most significant and discriminatory value. In our local clinical context, the ISS has proven to be of good value in predicting clinical outcomes (mortality). The initial resuscitation of the trauma patient, before arriving to the healthcare centers, affects the predictive value of RTSc and TRISS at the initial evaluation.

Keywords: Anatomic index, Mortality, Penetrating trauma, Physiologic index, Trauma severity index.

\section{INTRODUCTION}

El trauma es un importante problema de salud publica a nivel mundial debido a sus altas tasas de mortalidad, siendo responsable del $7-45 \%$ de las muertes en general y la principal causa de muerte en menores de 35 anios. $^{1-3}$ Desde los tiempos de guerra, diferentes sistemas de medida se desarrollaron con el fin de preparar al personal medico para el manejo oportuno del paciente

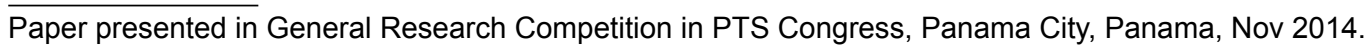


traumatizado, clasificandolo y pronosticando su desenlace. A traves del tiempo, multiples propuestas fueron evaluadas y llevadas a cabo, dando como resultado los sistemas de gravedad traumatica que son utilizados en la actualidad. ${ }^{2,4}$

Los indices de severidad del trauma (IST) son sistemas para evaluar, clasificar y codificar lesiones, que estan relacionados con caracteristicas especificas de cada victima y describen objetivamente su condicion, permitiendo que sean utilizados para evaluar pronostico. ${ }^{4}$ Sus principales funciones abarcan la prediccion del desenlace del trauma, la determinacion de la probabilidad de sobrevida, cuantificacion de las lesiones anatomicas y respuesta fisiologica de la victima, triage, comparacion de metodos terapeuticos, establecimiento de lineas de investigacion clinica y programas de control de calidad de la atencion medica en este grupo de pacientes. Por otra parte, un buen sistema de puntuacion debe cumplir 3 requerimientos para lograr estos objetivos: Precision, fiabilidad y especifidad, los cuales se han tratado de reunir en un indice universal, sin embargo este proposito no se ha logrado. ${ }^{2}$

Los IST se clasifican según las variables del paciente que cada uno utiliza, existen fisiologicos, anatomicos y mixtos. Las escalas fisiológicas mas comunes son: Revised trauma score (RTS) y Acute physiology and chronic health evaluation (APACHE). El RTS emplea 3 parametros fisiologicos: estado de consciencia (Puntaje en la escala de coma de Glasgow), presion arterial sistolica y frecuencia respiratoria, siendo el puntaje proporcional a la severidad del paciente y a su pronostico. Se han establecido como puntos de corte un RTS $<11$ y RTS corregido $<4$, con una prediccion de mortalidad mayor al 50\% e indicacion para remision a un centro especializado de trauma. ${ }^{2,5}$ La escala de APACHE, ha sido utilizada ampliamente en las unidades de cuidado intensivo, se basa en variables de comorbilidades cronicas y el componente fisiologico agudo, sin embargo tiene varias limitaciones en su aplicación a pacientes politraumatizados debido a que fue creada para otro tipo de poblacion $y$, necesita la recoleccion de varios datos y multiples calculos. ${ }^{2,5}$ Los indices anatomicos estan basadas en la descripcion de las heridas de las victima. El injury severity score (ISS) ha sido nombrado como estandar en la evaluacion del trauma, ${ }^{6}$ valora la severidad global del lesiones que sufre un paciente, basandose en el Abbreviated injury scale (AIS) que describe la gravedad de cada una de las 6 regiones corporales, proporcionandole un puntaje individual. El valor del ISS tiene una relacion proporcional con la severidad del trauma y la mortalidad, considerando un ISS > 25 trauma severo y, los cortes para sobrevida $95 \%$ y mortalidad del 95\%, un ISS < 15 e ISS > 40, respectivamente. La mayor limitacion que tiene este indice es la necesidad de clasificar quirurgicamente las lesiones, lo cual implica imposibilidad en su utilizacion como herramienta de triage y toma de decisiones en la practica inicial. ${ }^{2,7}$ Otra debilidad del ISS es la desestimacion de la severidad global de la victima por tener en cuenta una sola lesion de cada region corporal, dificultando la prediccion en pacientes con trauma multiple en una unica region. Por este motivo, los autores el ISS crearon un nuevo sistema de medicion similar, el New injury severity score (NISS) que empleaen su calculo las lesiones mas severas, independientemente de su localizacion, elevando su valor predictivo. $^{8}$ En el tercer grupo de esta clasificacion, se encuentra el Trauma and injury severity score (TRISS), calificado como un indice mixto debido a que combina parametros fisiologicos y anatomicos. Su utilizacion ha sido un éxito sobretodo en paises desarrollados, en los que ademas de predecir los desenlaces del paciente traumatizado, permite comparar y controlar los centros de atencion para estos pacientes. ${ }^{7}$ Todos los índices de severidad tienen ventajas y desventajas, cada Centro de Trauma en el mundo ha adoptado uno u otro según los estudios poblacionales regionales, pero hasta el momento ninguno ha sido avalado como el mejor.

\section{MATERIALES Y METODOS}

Se realizó un estudio prospectivo, observacional, de cohorte. Durante un periodo de 12 meses, mediante una historia clinica sistematizada, se captaron los pacientes que ingresaron al Hospital Universitario de Santander por trauma penetrante múltiple (compromiso de mas de una región corporal) y requirieron manejo quirúrgico. Se excluyeron pacientes con lesion de una sola region corporal aislada.

Se recopilo una muestra de 125 pacientes, se aplicaron los indices de severidad mas significativos (RTSc, ISS y TRISS) y se realizo seguimiento de los diferentes desenlaces hasta el momento del egreso del paciente. Las variables analizadas fueron relacionadas con caracteristicas sociodemograficas, del trauma, factores de riesgo, morbilidades, mortalidad y valores especificos de cada indice (Tabla 1). Se describieron estadisticamente cada una de las variables cuantitativas y, se describieron por medio de porcentaje y frecuencia cada una de las variables cualitativas. Ademas, se realizo la correlacion entre cada uno de los indices con algunas de las variables (estancia hospitalaria, estancia en UCI, UGRE), se evaluo el comportamiento de los indices de severidad respecto a los desenlaces mediante curvas de regresion logistica y se evaluo la prediccion de la mortalidad de cada uno de 
los incides, según la sensibilidad, especificidad y la curva ROC. Para lo anterior, se utilizaron el software SPSS y la herramienta Excel.

\section{Consideraciones Eticas}

En este estudio no se realizaron intervenciones o modificaciones de variables (estudio tipo observacional) por lo cual es catalogado como Investigacion sin riesgo según el Articulo 11 de la Resolucion No. 008430 de 1993. Los datos fueron recopilados de la historia clinica de trauma, formato que surgio de la historia clinica basica del paciente, se registro la informacion necesaria para obtener las variables previamente mencionadas, sin mostrar informacion sobre la identificacion de los pacientes, preservando la confidencialidad de cada uno. Por lo anterior, no se realizo consentimiento informado.

\section{RESULTADOS}

Este estudio se realizo basado en 125 pacientes captados, de los cuales 12 ingresaron a la institucion registadros con serial de paciente sin documento de identificacion y en el transcurso de su estancia hospitalaria, la informacion personal de los pacientes fue administrada por lo tanto los numeros de historias clinicas fueron reestablecidos, perdiendose la informacion completa del ingreso de cada uno. Adicionalmente, 2 pacientes fueron remitidos a Unidades de Cuidado Intensivo extrainstitucionales, lo cual no permitio completar el seguimiento de estos. Se siguieron 111 pacientes (Tabla 2), registrando datos hasta su egreso y se obtuvieron los siguientes resultados.

El $49.5 \%$ de los pacientes fueron trasladados a la institucion por medio de una ambulancia y el 50.5\%

Tabla 1: Clasificación de las variables utilizadas en el estudio

\begin{tabular}{ll}
\hline Variable & Tipo \\
\hline Edad & Cuantitativa \\
Género & Cualitativa \\
Estado civil & Cualitativa \\
Procedencia & Cualitativa \\
Transporte pre hospitalario & Cualitativa \\
Alcohol y/o Psicoactivos & Cualitativa \\
Tipo de herida & Cualitativa \\
Localización de las heridas & Cuantitativa \\
Control de daños & Cualitativa \\
Días de hospitalización & Cuantitativa \\
Días UCI & Cuantitativa \\
Unidad de glóbulos rojos (UGRE) & Cuantitativa \\
Número de reintervenciones & Cuantitativa \\
Muerte & Cualitativa \\
RTSc & Cuantitativa \\
TRISS & Cuantitativa \\
ISS & Cuantitativa \\
\hline
\end{tabular}

por medio de la policia $u$ otros medios de transporte particular/público. El 95\% de la poblacion procedía del area metropolitana de bucaramanga, con un $63.1 \%$ provinientes del casco urbano. Con lo anterior, se infiere que este porcentaje de pacientes recibieron reanimacion inicial en su sitio de remision.

\section{Características del Trauma}

La mayoria de las lesiones penetrantes fueron ocasionadas por arma cortopunzante (72\%) de las cuales $51.2 \%$ eran unicas y $48.7 \%$ eran multiples. Las heridas por arma de fuego correspondieron al $28 \%$ de los casos, siendo unicas en un $64.5 \%$ y multiples en $35.4 \%$.

Las regiones corporales mas frecuentemente lesionadas fueron torax y abdomen, 67 y $75 \%$ respectivamente, seguido de las extremidades y área general.

\section{Control de Dańos}

La cirugia de control de dańos se realizo en el 16.2\% de la poblacion, la mayoria de los casos por HPAF, con una mortalidad del 39\%, sin embargo, esta cifra fue variable debido a que solo el $61 \%$ de estos pacientes fueron trasladados a UCI (por escasa disponibilidad), aumentando la mortalidad en este grupo.

Se observó una correlacion significativa de los pacientes que fueron llevados a control de daños con el ISS, el $78 \%$ presentaron ISS $>25$. Por el contrario, la correlacion con RTSc fue baja, el $11 \%$ presentaron RTSc $<5$.

Tabla 2: Caracterízacion sociodemográfia de la población analizada en este estudio

\begin{tabular}{ll}
\hline Variable & Porcentaje \\
\hline Sexo & \\
Hombre & 91 \\
Mujer & 9 \\
Edad & \\
15-28 & 62 \\
$29-42$ & 22 \\
$43-57$ & 16 \\
Procedencia & \\
Bucaramanga y/o Área metropolitana & 95 \\
Otros municipios de Santander & 5 \\
Estado civil & \\
Soltero & 56.8 \\
Casado & 18 \\
Unión libre & 25.2 \\
Atención medica previa & \\
Si & 49.5 \\
No & 50.5 \\
Uso de alcohol/psicoactivos & \\
Alcohol & 34.2 \\
Psicoactivos & 0.9 \\
Ambas & 43.3 \\
Ninguna & 21.6 \\
\hline
\end{tabular}


Indices de Trauma: Prediccion del Desenlace

\section{Abdomen Abierto y Reintervenciones}

El uso del abdomen abierto y la necesidad de reintervenciones fueron poco comunes, 15.3 y $24 \%$ respectivamente. El rango de reintervenciones fue de 1 a 20 procedimientos, la mayoria de la muestra $(61.5 \%)$ demandó menos de dos y ambas variables se presentaron con mayor frecuencia en pacientes que ingresaron por HPAF. En cuanto a la correlacion entre los indices de severidad y estas dos variables, se encontraron valores significativos al comparar ISS - No. de reintervenciones, mientras que la correlacion con el RTS fue pobre (no significativa).

\section{Transfusion de Globulos Rojos}

El 65\% de los pacientes estudiados requirieron transfusion de unidades de globulos rojos empaquetados (UGRE), de los cuales el $59.5 \%$ fueron politransfusiones ( $>6$ UGRE) $\mathrm{y}$, se correlacionaron en un 66 y $26 \%$ con ISS $>25$ y RTS $<6$ respectivamente. Ambas fueron significativas estadisticamente, la correlacion RTS-UGRE fue negativa, indicando que por cada unidad de RTS se reduce la necesidad de transfusion de 0.8 UGRE. Por otro lado, la correlacion ISS-UGRE fue positiva, mostrando que el aumento de 1 unidad del ISS representa el aumento en la necesidad de transfusion de 0.21 UGRE.

\section{Estancia Hospitalaria}

El $68 \%$ de total permanecieron $<9$ dias hospitalizados, el $24 \%$ de $10-29$ dias y el $8 \%$ restante tuvieron hospitalizacion prolongada ( $>30$ dias). Se observó una correlacion positiva significativa entre los dias de hospitalizacion y el ISS, indicando que el aumento de una unidad ISS genera incremento de 0.67 dias de hospitalizacion, sin embargo, la correlacion con el RTS no generó una prediccion valida.

\section{Admision a Unidad de Cuidado Intensivo (UCI)}

Del grupo de pacientes estudiados, solo un pequeño porcentaje fueron admitidos a la UCI (14\%) debido a la poca disponibilidad en nuestro medio. El tiempo de estancia en la unidad varió desde 1 a 24 dias, sin embargo mas de la mitad de los pacientes estuvieron menos de 7 dias (56\%). Adicionalmente se observó que el 94\% de los admitidos presentaron ISS $>25$ y solo el $25 \%$ de estos presentaron RTSc $<6$. Lo anterior muestra una correlacion positiva significativa entre el ISS y la estancia en UCI, siendo un aumento de una unidad en el ISS indicativa de 0.25 dias de aumento en la estacia de UCI. La correlacion de esta variable con el RTSc no fue significativa.

\section{Mortalidad}

La mortalidad en la población estudiada fue de $9.9 \%$. Aproximadamente $2 / 3$ de los pacientes fallecidos ingresaron por heridas por arma de fuego y $1 / 3$ por heridas causadas por arma cortopunzante.

\section{Índices de Severidad}

Se analizaron los valores de los 3 índices de severidad más importantes y comúnmente usados (RTSc, ISS, TRISS). Según los análisis de sus valores y relaciones con las variables previamente descritas se obtuvieron los siguientes resultados.

\section{RTSc}

El valor promedio del RTSc en el total de la muestra fue de 7.24, valor máximo de 7.84, mínimo de 3.57 y con una desviación estándar de 1.05. Se observó que la mayor parte de los pacientes ingresaron estables hemodinamicamente y solo un $4 \%$ presento RTSc $<4$; por otro lado, al comparar los puntajes de los sobrevivientes vs fallecidos, no se encontró una diferencia significativa en los valores de este indice. Según el analisis estadistico, la curva ROC de este indice en HACP y en HPAF fue de 0.22 y 0.24 , respectivamente; la predicción del desenlace fue correcta en el $90.1 \%$, con punto de corte 5 , de la sobrevida en $98 \%$ y de la mortalidad en el $18.2 \%$, con punto de corte 4 .

\section{Triss}

El valor promedio del TRISS fue de 92, con un valor maximo de 99 , valor minimo de 15.9 y desviacion estandar de 13.7. El $86 \%$ de los pacientes tuvieron TRISS $>85$ y solo el $3 \%$ TRISS $<40$. De los pacientes que sobrevivieron, el $98 \%$ presentaron TRISS $>80$ y $2 \%$ TRISS $<40$, de los fallecidos, el $82 \%$ tenian valores mayores a 70 y el $18 \%$ menores a 40. La curva ROC de este indice en HACP y en HPAF fue de 0.14 y 0.19 , respectivamente; la prediccion del desenlace fue correcto en el $90.1 \%$ con punto de corte de 50 , de la sobrevida del $97 \%$ y de la mortalidad $18.2 \%$.

\section{ISS}

El valor promedio del ISS en el total de la muestra fue de 21.2, valor máximo de 43, mínimo de 9 y con una desviación estándar de 7.4. La mayor parte de la muestra presentó un rango entre 16-29 (67\%) y solo un $12 \%$ presento un ISS $>30$, adicionalmente, el $77 \%$ de los pacientes que sobrevivieron tenían ISS $<25$ (traumas leves - moderados), el 23\% un ISS > 25 (trauma severo), y de aquellos fallecidos, el $81 \%$ tuvieron valores superiores a 25 y solo el $18 \%$ inferiores a este, evidenciando una correlacion positiva significativa de este indice. La curva ROC de este indice en HACP y en HPAF fue de 0.68 y 0.73 , respectivamente; la predicción del desenlace en el grupo general de pacientes fue correcto en el $77.5 \%$ con punto de corte 25 , de la sobrevida en el $98 \%$ y de la mortalidad en 
el 81.8\%. Según lo anterior, el ISS fue un factor pronostico representativo para la prediccion de la mortalidad.

\section{Anexo}

Graficas representativas de las curvas roc para cada indice de severidad del trauma en la prediccion de mortalidad en pacientes con trauma penetrante multiple (Gráficos 1 to 6).

\section{CONCLUSIONES}

El trauma es un area de gran interes, principalmente para los medicos de urgencias y de las especialidades quirurgicas. En este estudio se evidenciaron varios aspectos importantes de los eventos traumaticos y la poblacion que los sufre, destacando que la mayoria de los pacientes son hombres, en edad reproductiva y activa economicamente; por otro lado, la mayor parte de estos pacientes ingresan bajo efecto de psicoactivos y alcohol, siendo frecuente el abuso de ambas sustancias y ademas, aproximadamente la mitad de la poblacion recibe atencion medica inicial de otras instituciones del

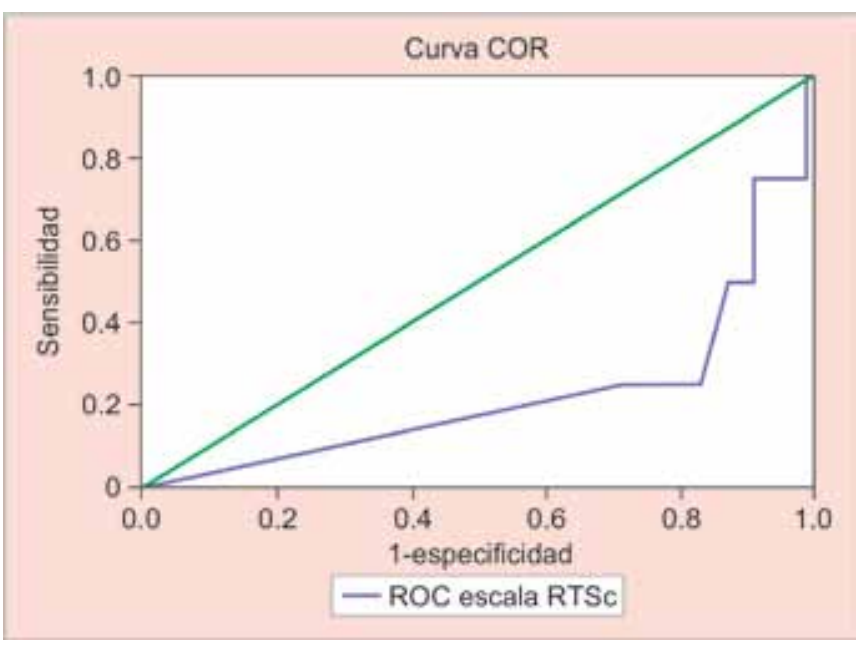

Gráfico 1: Análisis de la curva ROC para el RTSc en la prediccion de mortalidad en pacientes con HACP

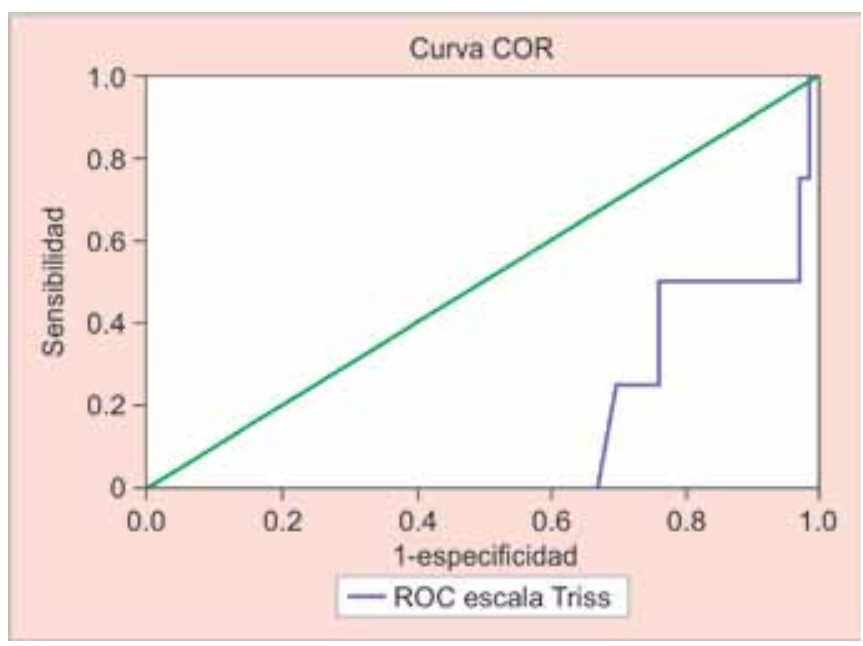

Gráfico 3: Análisis de la curva ROC para el TRISS en la prediccion de mortalidad en pacientes con HACP area metropolitana antes de ingresar al hospital de tercer nivel de complejidad (HUS).

El arma cortopunzante es la causa mas frecuente de trauma penetrante multiple en nuestro medio, siendo las toracoabdominales las que afectan mas frecuentemente dos o mas regiones corporales (41.3\%); adicionalmente, se evidenció un alto porcentaje de heridas por arma de fuego, que aun siendo unicas afectaron mas de una region corporal (64\%). En consecuencia de lo anterior, las dos regiones corporales mas comunmente comprometidas fueron torax (67\%) y abdomen (75\%).

La cirugia de control de daños se realizo en un porcentaje significativo, la mayoria de estos pacientes ingresaron por HPAF (72\%) de los cuales solo el $69 \%$ ingresaron a UCI, poniendo en evidencia la pobre oportunidad que tienen estos pacientes de acceder a este servicio en nuestro medio. Adicionalmente, se evidencio una diferencia significativa de la mortalidad entre el grupo de pacientes que ingresaron a UCI y el que no, lo cual indica que el manejo en dicha unidad

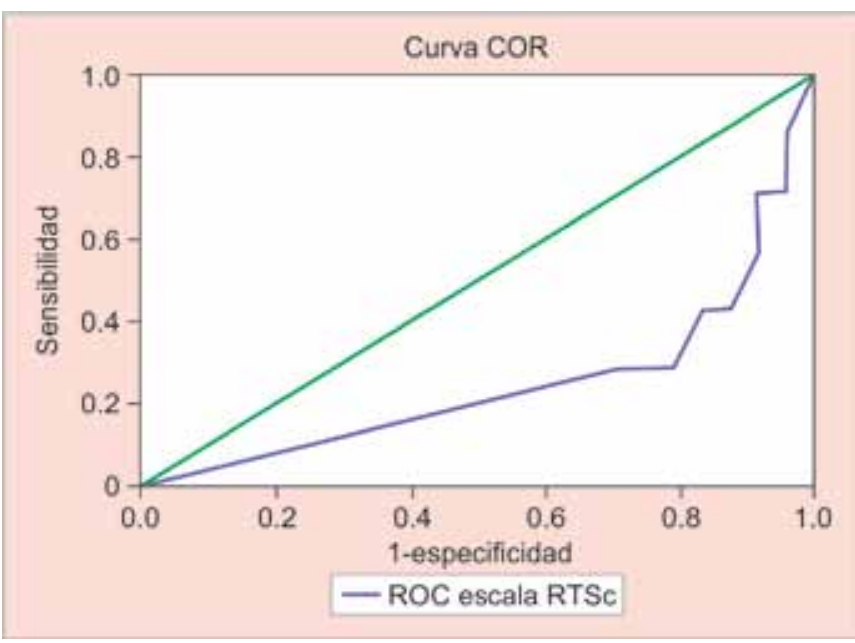

Gráfico 2: Análisis de la curva ROC para el RTSc en la prediccion de motalidad en pacientes con HPAF

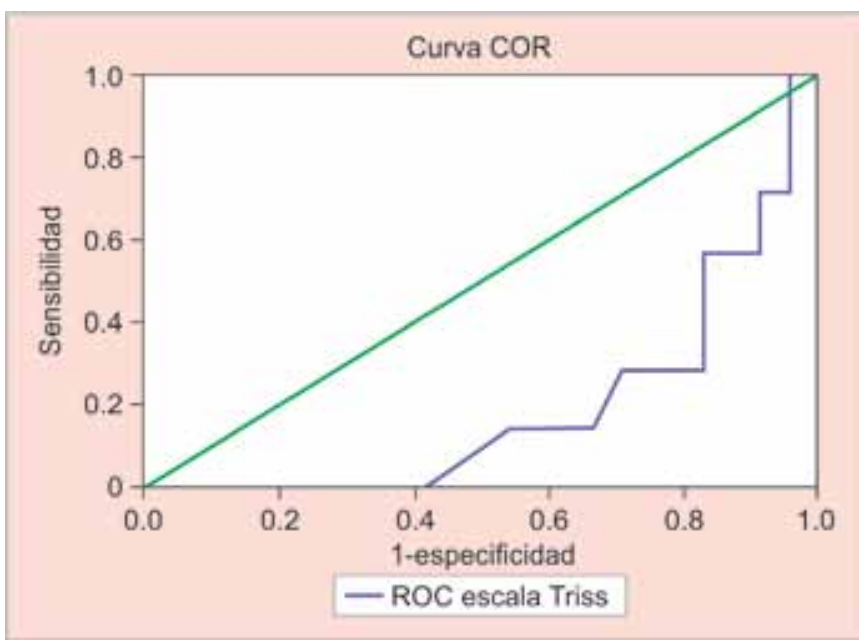

Gráfico 4: Análisis de la curva ROC para el en la prediccion de mortalidad en pacientes con HPAF 


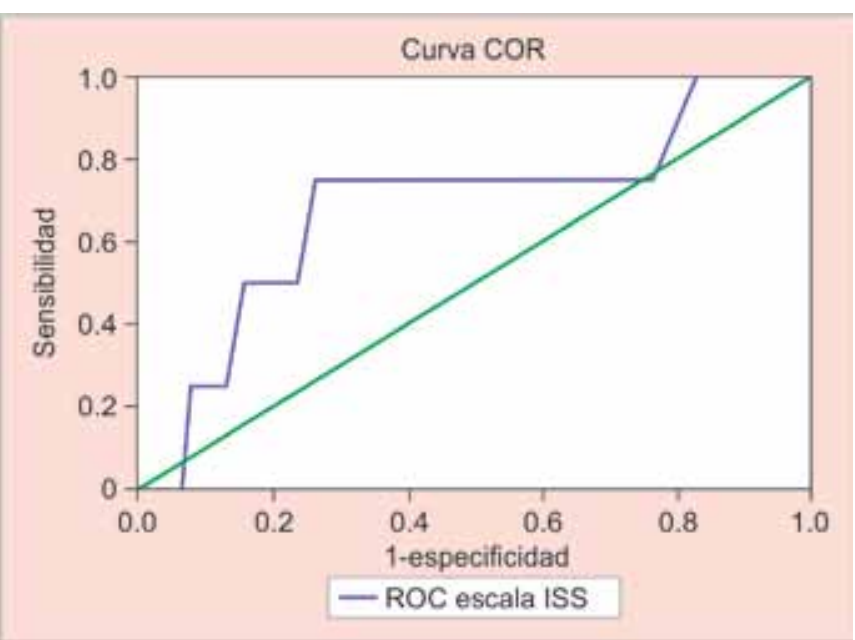

Gráfico 5: Análisis de la curva ROC para el ISS en la prediccion de mortalidad en pacientes con HACP

debe estar incluido para lograr un resultado optimo en estos pacientes. En cuanto al uso del abdomen abierto, se observó un bajo porcentaje, siendo mas frecuente en pacientes que habian sufrido heridas por arma de fuego.

Los indices de severidad que se analizaron en este estudio mostraron que el ISS fue el mejor predictor para la realizacion de la cirugia de control de daños en los pacientes con trauma penetrante multiple, usando un punto de corte de 25; sin embargo el RTSc tambien predijo correctamente la necesidad de realizar este procedimiento, usando un punto de corte de 4 . Asi mismo, estos dos indices se correlacionaron significativamente con la necesidad de transfusion de globulos rojos empaquetados, pero el ISS fue el unico que presento una relacion valida con el numero de reintervenciones, el tiempo de estancia hospitalaria y en la unidad de cuidado intensivo.

Los pacientes con trauma penetrante multiple presentaron una mortalidad mayor en los casos de heridas por arma de fuego. El ISS mostró el valor discrimitatorio mas aceptable y significativo, comportandose como un factor pronostico adecuado y adaptable en nuestro medio para la evaluacion de la mortalidad, mientras que el RTSc y el TRISS, no fueron factores pronosticos representativos. Lo anterior puede ser secundario al gran porcentaje de pacientes que ingresan remitidos de otra institucion en donde reciben atencion medica inicial (reanimacion inicial), generando un cambio significativo en los signos vitales al ingreso que no concuerdan con la severidad de las lesiones. Es de suma importancia tener en cuenta que los valores de la curva ROC obtenidos en el analisis del ISS fueron inferiores a los reportados en la literatura mundial $(>0.9)$, pero están en relación a los referidos en los estudios nacionales respecto al trauma penetrante (0.7).

Según lo anterior, se concluye que el componente anatomico es el factor pronostico mas confiable para la

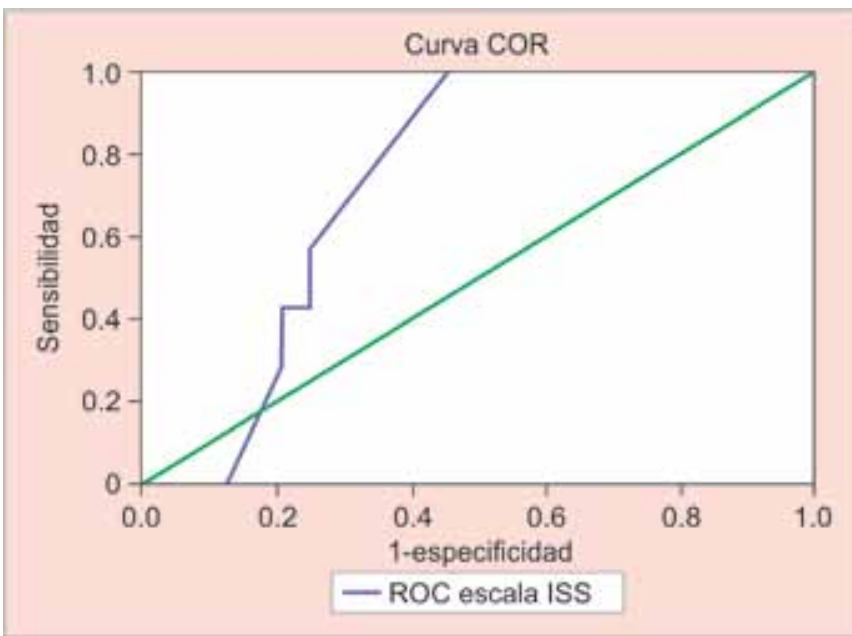

Gráfico 6: Análisis de la curva ROC para el ISS en la prediccion de mortalidad en pacientes con HPAF

prediccion de los desenlaces en los pacientes con trauma penetrante multiple de nuestro medio.

\section{LIMITACIONES DEL ESTUDIO}

Aunque los valores de la Curva ROC en el analisis del ISS y la prediccion de la severidad del trauma estuvieron por debajo del limite de la significancia estadistica (curva ROC >0.8), los resultados son equivalentes a los de estudios nacionales recientes ${ }^{8} \mathrm{y}$ muestran una prediccion adecuada de este indice. Lo anterior se debe posiblemente a un tamaño de muestra menor al necesario.

\section{REFERENCIAS}

1. Mattox KL, Moore EE, Feliciano DV. Trauma. 7th ed. New York: McGraw-Hill; 2012.

2. Chawda MN, Hildebrand F, Pape HC, Giannoudis PV. Predicting outcome after multiple trauma: which scoring system?. Injury Int J Care Injured 2004;35:347-358.

3. Guzzo JL, Bochicchio GV, Napolitano LM, Malone DL, Meyer W, Scalea TM. Prediction of Outcomes in Trauma: Anatomic or Physiologic Parameters?. J Am Coll Surg 2005;201:891-897.

4. Nogueira Lde S, Domingues Cde A, Campos Mde A, Sousa RM. Diez años del New Injury Severity Score (NISS): ¿cambio posible?. Rev Latino-am Enfermagem 2008;16(2):314-319.

5. Kuhls DA, Malone DL, McCarter RJ, Napolitano LM. Predictors of Mortality in Adult Trauma Patients: The Physiologic Trauma Score is equivalent to the Trauma and Injury Severity Score. J Am Coll Surg 2002 Jun;194(6):695-704.

6. Tamim H, Hazzouri AA, Mahfoud Z, El-Chemaly S. The injury severity score or the new injury severity score for predicting mortality, intensive care unit admission and length of hospital stay: Experience from a university hospital in a developing country. Injury Int J Care Injured 2008;39:115-120.

7. Athanassiadi K, Gerazounis M, Theakos N. Management of 150 flail chest injuries: analysis of risk factors affecting outcome. Eur J Cardiothorac Surg 2004;26(2):373-376.

8. Gélvez S, Ordóñez C. Evaluación de las escalas ISS y NISS en trauma penetrante grave. Rev Colomb Cir 2009;24:229-235. 


\section{Indices de Trauma: Prediccion del Desenlace}

Los autores presentan una población con características demográficas representativas de los pacientes traumatizados de todas las regiones de Colombia: hombres jóvenes que en un alto porcentaje tiene antecedentes de estar bajo los efectos de alcohol o agentes psicoactivos.

Hay que destacar desafortunadamente las condiciones de salud limitadas de la región por cuanto pacientes que requerían admisión a la UCI no dispusieron de este recurso lo cual aumenta el número de muertes.

Destacan una interesante correlación entre el ISS con la necesidad de realizar cirugía control de daños, número de reintervenciones, necesidad de transfundir glóbulos rojos, admisión a la unidad de cuidado intensivos y estancia hospitalaria; correlación no demostrada con el índice fisiológico RTS, esto deberá ser explicado probablemente por variables no incluidas en el análisis, la gravedad del trauma o el número de pacientes.

Las escalas de severidad del trauma que se utilizaron en esta investigación ayudan a las instituciones a evaluar la calidad en la atención que prestan a sus pacientes, su oportunidad y los recursos de que disponen para atenderlos. Es importante tener en cuenta que para hacer una buena estimación de estas escalas, la información debe registrarse en forma oportuna, clara y completa, con el fin de obtener una evaluación pertinente y real, pues será la única forma de que las instituciones reevalúen sus guías, protocolos o esquemas e implementen y mejoren grupos de respuesta rápida o unidades de trauma.

Los autores usaron la curva ROC como estrategia metodológica para evaluar modelos pronóstico; sirve como herramienta para discriminar o separar los individuos en dos grupos: sobrevive o muere (pronóstico). En la práctica clínica cotidiana la forma en que se interpreta es como la probabilidad de muerte dado un resultado de la escala RTS, ISS y TRISS. En el presente trabajo las escalas presentaron pobre desempeño con áreas bajo la curva inferiores a 0.7 lo que indica que no son buenas predictoras de mortalidad en la población estudiada. La capacidad de discriminación de las pruebas no puede evaluarse por cuanto no se presentan los intervalos de confianza correspondientes.

Debo estimular a los autores a continuar con esta línea de investigación que nos permita conocer, en una población con mayor número de casos, el comportamiento demográfico y clínico de los pacientes traumatizados y el desempeño de los puntajes de trauma en su población.

Carlos Hernando Morales Uribe Head, Department of Surgery University of Antioquia Medellin, Colombia 


\section{INVITED COMMENTARY}

\section{Indices of Trauma: Prediction of the Outcome}

The study sample is representative of traumatized patients in all the regions of Colombia: Which usually are young men, frequently under the influence of alcohol or other substances.

Unfortunately it is important to highlight the limited health resources available in the region, specially the lack of an ICU facility. Patients that fulfilled criteria for an ICU admission could not access this resource, and this implied an increase in mortalities.

They highlight an interesting correlation between the ISS with the need for damage control surgery, number of surgical interventions, need for blood transfusions, admission to ICU and length of hospital stay. This association was not found with the RTS physiologic index, a conclusion that should be explained by variables not included in the study, trauma severity or number of patients.

The trauma severity scores used in this study help to evaluate the institutions, specially the quality of care, availability of resources, and the use and timing of the latter. It is important to clarify that to use these scales correctly the data must be registered in a complete, clear and timely fashion, with the objective of obtaining real and pertinent information. This is the only way in which institutions can re-evaluate their guidelines, protocols and treatment algorithms to create a fruitful quality improvement initiative for their acute care surgery models.

The authors used a ROC curve as a methodological strategy to evaluate prognostic models, which are useful as a tool to identify individuals in two groups: High probability of survival or high-risk mortality. In everyday practice, this is interpreted as the probability of dying based on a RTS, ISS or TRISS result. In this study, the scales had a poor performance with AUC inferior to 0.7 , which suggests that they are not good mortality predictors. The capacity of these tools to discriminate the possible results cannot be analyzed, as there are no confidence intervals presented.

I encourage the authors to continue on this line of investigation, in order to identify, in a study with a greater sample size, the demographics and natural history of traumatized patients, along with the performance of these trauma scales in identifying patients with a higher mortality risk.

Carlos Hernando Morales Uribe Head, Department of Surgery University of Antioquia Medellin, Colombia 